\title{
Average Channel Capacity of LTE Downlink Multiuser MIMO Systems
}

\author{
Pei Xiao ${ }^{\dagger}$, Zihuai Lin ${ }^{\dagger \dagger}$, Colin Cowan ${ }^{\dagger}$ \\ ${ }^{\dagger}$ Queen's University Belfast, BT3 9DT, United Kingdom \\ E-mail: \{pei.xiao,c.cowan\}@ecit.qub.ac.uk \\ ${ }^{\dagger \dagger}$ University of Sydney, 2006 Sydney, Australia \\ E-mail: zihuai@ee.usyd.edu.au
}

\begin{abstract}
The average channel capacity for multiuser Multiple Input Multiple Output (MIMO) systems is analyzed in this paper. The analysis model is based on the generalized 3GPP LTE downlink transmission for which two Spatial Division Multiplexing (SDM) multiuser MIMO schemes are investigated: Single User (SU) and Multi-user (MU) MIMO schemes. The packet scheduler is used to exploit the available multiuser diversity in all the three physical domains (i.e., space, time and frequency). The main contribution of this paper is the establishment of a mathematical model for the channel capacity of multiuser SDM MIMO systems with frequency domain packet scheduler.
\end{abstract}

Keywords: Channel capacity, LTE, OFDMA, Multiuser MIMO.

\section{INTRODUCTION}

In 3GPP Long Term Evolution (LTE) (also known as Evolved-UMTS Terrestrial Radio Access (E-UTRA)), MultipleInput Multiple-Output (MIMO) and Orthogonal Frequency Division Multiple Access (OFDMA) have been selected for downlink transmission [1]. Both Spatial Division Multiplexing (SDM) and Frequency Domain Packet Scheduling (FDPS) have been proposed. SDM is used to improve the spectral efficiency of the system, while FDPS allows the packet scheduler at the Base Station (BS) to exploit the available multiuser diversity in both time and frequency domain. In [2], it is shown that the MIMO schemes with combined SDM and FDPS can further enhance the system performance.

Both open loop and closed loop $\mathrm{MIMO}^{1}$ are considered as possible solutions in 3GPP LTE. However, the use of closed loop provides both diversity and array gains, and hence a superior performance. Due to its simplicity and robust performance, the use of linear precoding has been widely studied as a closed loop scheme [2]. Here, we refer to the open loop MIMO as the SDM MIMO without precoding, and the closed loop MIMO as the linearly precoded SDM MIMO. Most of the existing work on linear precoding focuses on the design of the transmitter precoding matrix, e.g., [3], [4]. In [5], [6], the interaction between packet scheduling and array antenna techniques is studied based on a system level simulation model. The interactions between multiuser diversity and spatial diversity is investigated analytically in [7], with the focus on space time block coding. To the authors knowledge, theoretical analysis of linearly precoded multiuser SDM MIMO systems combined with FDPS has not been studied so far. In this paper, we conduct a theoretical analysis for the average

\footnotetext{
${ }^{1}$ Open loop and closed loop MIMO correspond to the MIMO systems withou and with channel state information at the transmitter, respectively [1]
}

channel capacity in multiuser MIMO systems with SDM-FDPS Although our study is conducted for the 3GPP LTE downlink packet data transmission [1], the analysis method is generally applicable to other packet switched systems.

\section{SYSTEM MODEL}

In this section, we describe the system model of multiuser SDM MIMO schemes for 3GPP LTE downlink transmission with packet scheduling. The basic scheduling unit in LTE is the Physical Resource Block (PRB), which consists of a number of consecutive OFDM sub-carriers reserved during the transmission of a fixed number of OFDM symbols. One PRB of 12 contiguous subcarriers can be configured for localized transmission $^{2}$ in a sub-frame. With the localized transmission scheme, two SDM schemes are now under investigation [1], i.e., Single User (SU) MIMO and Multi-User (MU) MIMO schemes. They differ in terms of the freedom allowed to the scheduler in the spatial domain [1]. With SU-MIMO scheme, only one single user can be scheduled per PRB; whereas with MU-MIMO scheme, multiple users can be scheduled per PRB, one user for each sub-stream per PRB.

The Frequency Domain (FD) scheduling algorithm considered in this work is the FD Proportional Fair (PF) [8] packet scheduling algorithm, which is being investigated under LTE. It is shown in [9] that a scheduler is PF if the instantaneous rate $\left\{R_{k, i}\right\}$ maximizes $\left[\frac{R_{k, i}}{\overline{R_{k, i}}}\right]$, where $\overline{R_{k, i}}=\left(1-\frac{1}{T_{w i n}}\right) \overline{R_{k-1, i}}+$ $\frac{1}{T_{w i n}} R_{k, i}$ is the moving average of the maximum achievable rate of user $i$ at the $k$ th time slot over a sliding window of $T_{\text {win }}$ time slots.

The system considered here has $n_{t}$ transmit antennas at the Base Station (BS) and $n_{r}^{i}$ receive antennas at the $i$ th Mobile Station (MS), $i=1,2, \cdots, K$. Without loss of generality, we assume that all the MSs have equal numbers of antennas $n_{r}$, and define $M=\min \left(n_{t}, n_{r}\right)$ and $N=\max \left(n_{t}, n_{r}\right)$. The number of users simultaneously served on each PRB for the MU-MIMO scheme is usually limited by the number of transmitter antennas $n_{t}$. The scheduler in BS select at most $n_{t}$ users per PRB from the $K$ active users in the cell for data transmission. Denote by $\zeta_{k}$ the set of users scheduled on the $k_{t h} \mathrm{PRB}$ and $\left|\zeta_{\mathbf{k}}\right|=n_{t}$. Without precoding, the received signal vector at the $j$ th $\mathrm{MS}$, $j \in \zeta_{n}$, can be modeled as $\mathbf{y}_{n, j}=\mathbf{H}_{n, j} \mathbf{x}_{n}+\mathbf{n}_{n, j}$, where $\mathbf{n}_{n, j} \in \mathbb{C}^{n_{r} \times 1}$ is a circularly symmetric complex Gaussian noise

\footnotetext{
${ }^{2}$ In the localized FDMA transmission scheme, each user's data is transmitted by consecutive subcarriers, while for the distributed FDMA transmission 1 scheme, the user's data is transmitted by distributed subcarriers [1].
} 
vector with a zero mean and covariance matrix $N_{0} \mathbf{I} \in \mathbb{R}^{n_{r} \times n_{r}}$, i.e., $\mathbf{n}_{n, j} \sim \mathcal{C N}\left(\mathbf{0}, N_{0} \mathbf{I}\right) ; \mathbf{H}_{n, j} \in \mathbb{C}^{n_{r} \times n_{t}}$ is the channel matrix between the BS and the $j$ th MS at the $n$th PRB and $\mathbf{x}_{n} \in \mathbb{C}^{n_{t} \times 1}$ is the transmitted signal vector at the $n$th PRB, and the $\mu$ th element of $\mathbf{x}_{n}$ is the data symbol $x_{n, \mu}$ transmitted from the $\mu$ th MS, $\mu \in \zeta_{n}$.

For a linearly precoded MIMO system, the received signal vector at the $j$ th MS, $j \in \zeta_{n}$, can be formed as

$\mathbf{y}_{n, j}=\mathbf{H}_{n, j} \sum_{\mu \in \zeta_{n}} \mathbf{b}_{n, \mu} x_{n, \mu}+\mathbf{n}_{n, j}=\mathbf{H}_{n, j} \sum_{\mu \in \zeta_{n}} \mathbf{B}_{n, \mu} \boldsymbol{\psi}_{n, \mu}+\mathbf{n}_{n, j}$,

where $\mathbf{b}_{n, \mu} \in \mathbb{C}^{n_{t} \times 1}$ is the beamvector for the $\mu$ th MS user data on the $n$th PRB and $\mathbf{B}_{n, \mu} \in \mathbb{C}^{n_{t} \times n_{t}}$ is the precoding matrix with the $\mu$ th column of $\mathbf{B}_{n, \mu}$ equal to $\mathbf{b}_{n, \mu}, \boldsymbol{\psi}_{n, \mu} \in \mathbb{C}^{n_{t} \times 1}$ is a column vector in which the $\mu$ th element equal to $x_{n, \mu}$ and the rest equal to zero.

For MU-MIMO SDM scheme, we assumer the MS only report quantized channel state information to the BS. The BS select the users with the same quantized channel state information for MIMO transimission. In this case, the precoding matrices for the selected users will be the same, therefore, the received signal at the jth MS becomes

$$
\mathbf{y}_{n, j}=\mathbf{H}_{n, j} \mathbf{B}_{n, \mu} \sum_{\mu \in \zeta_{n}} \boldsymbol{\psi}_{n, \mu}+\mathbf{n}_{n, j}=\mathbf{H}_{n, j} \mathbf{B}_{n, \mu} \mathbf{x}_{n}+\mathbf{n}_{n, j},
$$

where $\mathbf{B}_{n, \mu}$ is the precoding matrix with the $\mu$ th column equal to $\mathbf{b}_{n, \mu}$ on the $n$th PRB, $\mu \in \zeta_{n}$.

With a linear Minimum Mean Square Error (MMSE) receiver, also known as a Wiener filter, the optimum precoding matrix under the sum power constraint can be generally expressed as $\mathbf{B}_{n, j}=\mathbf{U}_{n, j} \sqrt{\boldsymbol{\Sigma}_{n, j}} \mathbf{V}_{n, j}$ [10]. Here $\mathbf{U}_{n, j}$ is an $n_{t} \times n_{t}$ eigenvector matrix with columns corresponding to the $n_{t}$ largest eigenvalues of the matrix $\mathbf{H}^{0}{ }_{n, j} \mathbf{H}_{n, j}^{0}$, where $\mathbf{H}_{n, j}^{0^{H}}$ is the Hermitian transpose of the quanized channel matrix $\mathbf{H}_{n, j}^{0}$. For Schur-Concave objective functions, $\mathbf{V}_{n, j} \in \mathbb{C}^{n_{t} \times n_{t}}$ is an unitary matrix, and $\boldsymbol{\Sigma}_{n, j}$ is a diagonal matrix with the $\eta$ th diagonal entry $\boldsymbol{\Sigma}_{n, j}(\eta, \eta)$ representing the power allocated to the $\eta$ th established data sub-stream, $\eta \in\left\{1,2, \cdots, n_{t}\right\}$.

\section{THE AVERAGE CHANNEL CAPACITY}

The average channel capacity [11] or the so called Shannon (Ergodic) Capacity [12] per PRB can be obtained by

$$
C=\int_{0}^{\infty} \log _{2}(1+\gamma) f_{\Gamma}(\gamma) d \gamma
$$

where $f_{\Gamma}(\gamma)$ is the probability density function (PDF) of $\gamma$, the effective signal to interference plus noise ratio (SINR).

With the investigated linear receivers, which decompose the MIMO channel into independent channels, the total capacity for the multiple input sub-stream MIMO systems is equal to the sum of the capacities for each sub-stream, i.e.,

$$
C_{\text {total }}=\sum_{i=0}^{Q-1} \int_{0}^{\infty} \log _{2}(1+\gamma) f_{\Gamma_{i}}(\gamma) d \gamma
$$

where $Q$ is the number of sub-streams.

\section{A. Average channel capacity for SDM MIMO without precoding}

The average channel capacity for SDM SU-MIMO without precoding can be obtained as

$$
\begin{aligned}
C_{S U}^{O} & =\int_{0}^{\infty} d \gamma \log _{2}(1+\gamma) K \frac{4 e^{4 / \gamma_{0}}}{\gamma_{0}^{2}}\left[\gamma_{0}^{2}(1+\gamma)\right]^{-\frac{1}{4}} \\
& \cdot \exp \left(-\frac{4}{\gamma_{0}} \sqrt{1+\gamma}\right) \sqrt{\frac{\pi}{2}} \gamma_{0} \sum_{n=0}^{\infty} \frac{(1 / 2-n)^{2 n}}{2^{n / 2}\left(\frac{4}{\gamma_{0}} \sqrt{1+\gamma}\right)^{n}} \\
& \cdot\left[1-e^{-\frac{2 \gamma}{\gamma_{0}}}-2 e^{4 / \gamma_{0}} \int_{\gamma_{0}}^{\gamma_{0}(1+\gamma)} e^{-2 / \gamma_{0}^{2} u-2(1+\gamma) u^{-1}} d u\right]^{K-1},
\end{aligned}
$$

where $K$ is the number of active users in the cell or the so called User Diversity Order (UDO); $\gamma_{0}=E_{s} / N_{0}, E_{s}$ is the average transmit symbol energy per antenna and $N_{0}$ is the power spectral density of the additive white Gaussian noise. The derivation of (5) is given in Appendix A.

For SDM MU-MIMO without precoding, the PDF for the post scheduling sub-stream SINR can be derived as [13]

$$
f_{\Gamma_{k}}^{M s}(\gamma)=\frac{d}{d \gamma} F_{\Gamma_{k}}^{M s}(\gamma)=\frac{n_{t}}{\gamma_{0}} e^{-\frac{n_{t} \gamma}{\gamma_{0}}} K\left(1-e^{-\frac{n_{t} \gamma}{\gamma_{0}}}\right)^{(K-1)} .
$$

The average channel capacity of SDM MU-MIMO without precoding is the sum of the average channel capacity for each sub-stream. Substituting the PDF for the post scheduling substream SINR expressed by (6) into (4) yields

$$
\begin{aligned}
C_{M U}^{O} & =\frac{n_{t} K}{\gamma_{0}} \sum_{i} \int_{0}^{\infty} \log _{2}(1+x) e^{-\frac{n_{t} x}{\gamma_{0}}}\left(1-e^{-\frac{n_{t} x}{\gamma_{0}}}\right)^{K-1} d x \\
& =\frac{n_{t} K}{\gamma_{0} \ln 2} \sum_{i} \sum_{j=0}^{K-1}(-1)^{j}\left(\begin{array}{c}
K-1 \\
j
\end{array}\right) \frac{e^{-a_{j}} E_{i}\left(a_{j}\right)}{a_{j}}
\end{aligned}
$$

where $a_{j}=-\frac{(j+1) n_{t}}{\gamma_{0}}$, and $E_{i}(\cdot)$ is the exponential integral function defined as [16, p. 875-877]

$$
E_{i}(x)=\int_{-\infty}^{x} \frac{e^{t}}{t} d t=\ln (-x)+\sum_{m=1}^{\infty} \frac{x^{m}}{m \cdot m !} \quad x<0 .
$$

The derivation of (7) is given in Appendix B.

\section{B. Average channel capacity for SDM MIMO with precoding}

For a linearly precoded multiuser SDM SU-MIMO scheme with FDPS, assuming 2 antennas at both the transmitter and the receiver, the probability density function of the effective SINR can be expressed as [13]

$$
\begin{aligned}
& f_{\Gamma_{u}}^{C S}(\gamma)=K \int_{0}^{\gamma} \frac{1}{\left(\rho_{1} \rho_{2}\right)^{3}(1+v)} \exp \left(-\frac{v}{\rho_{1}}-\frac{\gamma-v}{\rho_{2}(1+v)}\right) \\
& \cdot\left(\rho_{2} v-\frac{\gamma-v}{1+v} \rho_{1}\right)^{2} d v\left(\int_{0}^{\gamma} \frac{1}{\left(\rho_{1} \rho_{2}\right)^{3}} \exp \left(-\frac{v}{\rho_{1}}\right) \varphi(\gamma, v) d v\right)^{K-1}
\end{aligned}
$$

where $\rho_{j}=p_{j} / N_{0}(j=1,2)$, and $p_{j}$ is the power allocated to 2 the $j$ th established sub-stream of the $i$ th MS. Substituting (9) 
into (3) yields the post scheduling average channel capacity of a linearly precoded SDM SU-MIMO scheme

$C_{S U}^{C}=\int_{0}^{\infty} d \gamma \log _{2}(1+\gamma) K\left(\int_{0}^{\gamma} \frac{1}{\left(\rho_{1} \rho_{2}\right)^{3}} \exp \left(-\frac{v}{\rho_{1}}\right) \varphi(\gamma, v) d v\right)$

$\cdot \int_{0}^{\gamma} d v \frac{1}{\left(\rho_{1} \rho_{2}\right)^{3}(1+v)} \cdot \exp \left(-\frac{v}{\rho_{1}}-\frac{\gamma-v}{\rho_{2}(1+v)}\right)\left(\rho_{2} v-\frac{\gamma-v}{1+v} \rho_{1}\right)^{2}$.

According to [13], the PDF of the instantaneous SINR of the $i$ th sub-stream of each PRB with linearly precoded MU-MIMO scheme using FD PF packet scheduling algorithm can then be obtained as

$$
\begin{aligned}
& f_{\Gamma_{i}}^{C M}(\gamma)= \frac{d}{d \gamma} F_{\Gamma_{i}(\gamma)}^{C M} \simeq \frac{K\left(\gamma / \rho_{i}\right)^{\beta(i)-1}}{\rho_{i}[\beta(i)-1] ! \tilde{\lambda}_{i}^{\beta(i)}} \exp \left(-\gamma /\left(\rho_{i} \tilde{\lambda}_{i}\right)\right) \\
& \cdot\left[1-\sum_{j=0}^{\beta(i)-1} \frac{\left(\gamma /\left(\rho_{i} \tilde{\lambda}_{i}\right)\right)^{j}}{j !} \exp \left(-\gamma /\left(\rho_{i} \tilde{\lambda}_{i}\right)\right)\right]^{K-1}
\end{aligned}
$$

where $\beta(i)=\left(n_{t}-i+1\right)\left(n_{r}-i+1\right)$ and $\tilde{\lambda}_{i}=\frac{1}{\beta(i)} \bar{\lambda}_{i}=$ $\frac{1}{\beta(i)} \int_{0}^{\infty} \lambda_{i} f_{\Lambda}\left(\lambda_{i}\right) d \lambda_{i}, f_{\Lambda_{i}}\left(\lambda_{i}\right)$ is the marginal PDF of $\lambda_{i}$. Substituting (11) into (4) yields the average channel capacity of the linearly precoded multiuser SDM MU-MIMO scheme

$$
\begin{aligned}
& C_{M U}^{C}=\frac{K}{\left(\rho_{1} \tilde{\lambda}_{1}\right)^{\beta(i)}(\beta(i)-1) !} \\
& \underbrace{\int_{0}^{\infty} \log _{2}(1+\gamma) \underbrace{\gamma^{\beta(i)-1} e^{-\frac{\gamma}{\rho_{1} \tilde{\lambda}_{1}}}\left[1-\sum_{j=0}^{\beta(i)-1} \frac{\gamma^{j}}{j !\left(\rho_{1} \tilde{\lambda}_{1}\right)^{j}} e^{-\frac{\gamma}{\rho_{1} \tilde{\lambda}_{1}}}\right]^{K-1}}_{\boldsymbol{\Psi}(\gamma)} d \gamma}_{\boldsymbol{\Omega}} \\
& +\frac{K}{\rho_{2} \tilde{\lambda}_{2}} \underbrace{\int_{0}^{\infty} \log _{2}(1+\gamma) e^{-\frac{\gamma}{\rho_{2} \tilde{\lambda}_{2}}}\left(1-e^{-\frac{\gamma}{\rho_{2} \tilde{\lambda}_{2}}}\right)^{K-1} d \gamma}_{\boldsymbol{\Phi}} .
\end{aligned}
$$

Following the same procedure as shown in Section III-A for SDM MU-MIMO without precoding, we have

$$
\boldsymbol{\Phi}=\frac{1}{\ln 2} \sum_{j=0}^{K-1}(-1)^{j}\left(\begin{array}{c}
K-1 \\
j
\end{array}\right) \frac{e^{-b_{j}} E_{i}\left(b_{j}\right)}{b_{j}},
$$

where $b_{j}=-\frac{(j+1)}{\rho_{2} \lambda_{2}}$. With binomial expansion, we obtain

$$
\begin{aligned}
\Psi(\gamma)= & \gamma^{\beta(i)-1} \sum_{n=0}^{K-1}(-1)^{n} \frac{(K-1) !}{(K-1-n) ! n !} \\
& \cdot\left(\sum_{j=0}^{\beta(i)-1} \frac{\gamma^{j}}{j !\left(\rho_{1} \tilde{\lambda}_{1}\right)^{j}}\right)^{n} e^{-\frac{(n+1) \gamma}{\rho_{1} \tilde{\lambda}_{1}}} \\
= & \gamma^{\beta(i)-1} \sum_{n=0}^{K-1} c_{n}\left(\sum_{j=0}^{\beta(i)-1} \frac{\gamma^{j}}{j !\left(\rho_{1} \tilde{\lambda}_{1}\right)^{j}}\right)^{n} e^{-\frac{(n+1) \gamma}{\rho_{1} \tilde{\lambda}_{1}}}
\end{aligned}
$$

where

$$
c_{n}=(-1)^{n} \frac{(K-1) !}{(K-1-n) ! n !}=(-1)^{n}\left(\begin{array}{c}
K-1 \\
n
\end{array}\right) .
$$

According to (12), $\boldsymbol{\Omega}=\int_{0}^{\infty} \log _{2}(1+\gamma) \boldsymbol{\Psi}(\gamma) d \gamma$.
For a large number of transmit and receiver antennas (assume $\eta=n_{t}=n_{r}$ and the ordered eigenvalues of $\mathbf{H H}^{H}$ as $\lambda_{1} \geq$ $\left.-1 \lambda_{2} \geq \cdots \geq \lambda_{\eta}\right)$, i.e., $\beta(i)$ is sufficiently large, the average channel capacity for SDM MU-MIMO with precoding can be approximated by a closed form

$$
\begin{aligned}
C_{M U}^{C} \approx & \sum_{i=1}^{\eta-1} \frac{K}{\left(\rho_{i} \lambda_{i}\right)^{\beta(i)-1}(\beta(i)-1) !} \frac{1}{\ln 2} \\
& \cdot \sum_{n=0}^{K-1}(-1)^{n} \frac{(K-1) !}{(K-1-n) ! n !} \mathcal{I}_{\beta(i)}\left(\frac{1}{\rho_{i} \lambda_{i}}\right) \\
+ & \frac{K}{\rho_{\eta} \tilde{\lambda}_{\eta}} \frac{1}{\ln 2} \sum_{j=0}^{K-1}(-1)^{j}\left(\begin{array}{c}
K-1 \\
j
\end{array}\right) \frac{e^{-d_{j}} E_{i}\left(d_{j}\right)}{d_{j}}
\end{aligned}
$$

where $d_{j}=-\frac{(j+1)}{\rho_{\eta} \lambda_{\eta}}$. The function $\mathcal{I}(\cdot)$ is defined as [14]

$$
\begin{aligned}
\mathcal{I}_{i}(\mu) & =\int_{0}^{\infty} \ln (1+x) x^{i-1} e^{-\mu x} d x, \quad \mu>0, i=1,2, \ldots \\
& =(i-1) ! e^{\mu} \sum_{k=1}^{i} \frac{\Gamma(-i+k, \mu)}{\mu^{k}},
\end{aligned}
$$

and $\Gamma(\cdot, \cdot)$ is the complementary incomplete gamma function defined as [14]

$$
\Gamma(\alpha, x)=\int_{x}^{\infty} t^{\alpha-1} e^{-t} d t
$$

The derivation of (16) is given in Appendix C.

\section{Analytical Results}

We consider the case with 2 antennas at both the transmitter and the receiver side. The average channel capacity for SU and MU MIMO schemes versus transmitted SNR and the number of active users are shown in Fig. 1 and Fig. 2, respectively.

Fig. 1 indicates that in a cell with 10 active users, the MUMIMO schemes (no matter with or without precoding) always outperform the SU-MIMO schemes. Note that the performance for the closed loop SU-MIMO with precoding in Fig. 1 is slightly worse than the one for the open loop MU-MIMO. This implies that MU-MIMO exploits more multiuser diversity gain than SU-MIMO does. Interestingly, the precoding gain for SUMIMO is much larger than for MU-MIMO.

Fig. 2 shows the average channel capacity for SU and MU MIMO schemes versus the number of active users in the cell. Both the simulation results and the analytical results for the open loop and the linearly precoded MIMO systems are shown. It can be seen that the simulation results almost coincide with the analytical results. Fig. 2 indicates that in a cell with 10 active users, the MU-MIMO schemes (no matter with or without precoding) always perform better than the SU-MIMO schemes. Interestingly, the precoding gain for SU-MIMO is much larger than for MU-MIMO. Fig. 2 shows that the average channel capacity for SU-MIMO schemes with precoding is always higher than the one for the SU-MIMO scheme without precoding regardless of the number of users. However, for the MU-MIMO scheme, the above observation does not hold especially for systems with a large number of active users. As 3 the number of active users increases, the advantages of using 
precoding schemes gradually vanish. This can be explained by the fact that the multiuser diversity gain has already been exploited by MU-MIMO schemes and the additional diversity gain by using precoding does not contribute too much in this case. Note that we used ZF receiver for the open loop scheme while for the closed loop scheme, the MMSE receiver was employed. One reason why we use ZF receiver instead of MMSE for the open loop scheme is that the SINR distribution for the open loop scheme with MMSE receiver is very difficult to obtain. Another reason is that the ZF receiver can separate the received data sub-streams, while MMSE receiver can not, the independence property of the received data sub-streams is used for computing the effective SINR as mentioned earlier.

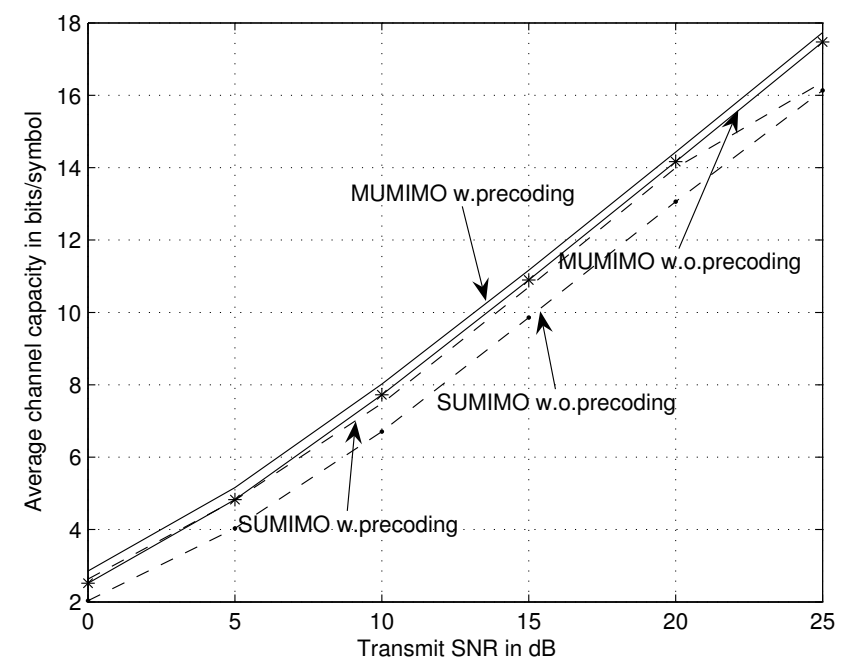

Fig. 1. Average channel capacity vs. transmit SNR for multiuser SU and MU MIMO schemes with/without linear precoding, number of active users is 10 .

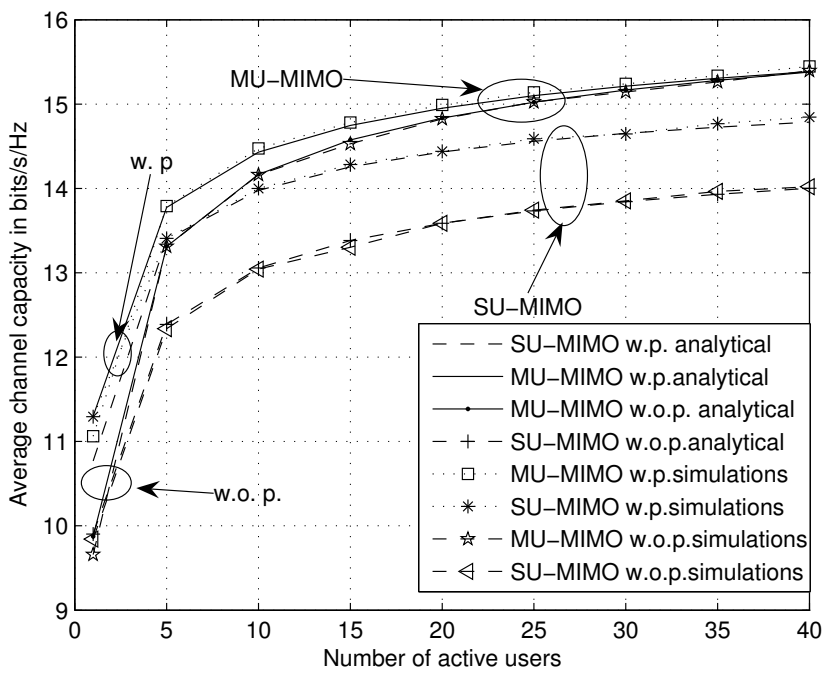

Fig. 2. Average channel capacity vs. number of active users for multiuser SU and MU MIMO schemes with/without linear precoding, transmit SNR is $20 \mathrm{~dB}$. "w.p" represents "with precoding", "w.o.p." represents "without precoding".

\section{CONCLUSION}

In this paper, we analyzed the average channel capacity of the LTE downlink multiuser systems with linearly precoded SDM MIMO schemes in conjunction with a base station packet scheduler. Both SU and MU MIMO schemes with FDPS are investigated. Our analysis reveals that the system using a linearly precoded MU-MIMO scheme has a larger capacity than the one using a SU-MIMO scheme. For a SU-MIMO scheme, the precoded MIMO system always has a higher average channel capacity than the one without precoding. For a MU-MIMO, the above conclusion does not hold, particularly for systems with a large number of active users. The analysis conducted in this paper provides a theoretical reference for the practical implementation of the LTE systems.

\section{APPENDIX}

A. Derivation of (5) - the average channel capacity for $S U$ MIMO without precoding

The PDF of the post scheduling effective SINR for SDM SU-MIMO without precoding has been derived in [13] as

$$
\begin{gathered}
f_{\Gamma_{u}}^{O S}(\gamma)=K\left[\int_{0}^{\gamma} \frac{2}{\gamma_{0}} e^{-\frac{2 x}{\gamma_{0}}}\left(1-e^{-\frac{2(\gamma-x)}{\gamma_{0}(1+x)}}\right) d x\right]^{K-1} \\
\cdot \int_{0}^{\gamma}\left[\frac{4}{\gamma_{0}^{2}(1+x)} \exp \left(-\frac{2\left(\gamma+x^{2}\right)}{\gamma_{0}(1+x)}\right)\right] d x .
\end{gathered}
$$

Substituting (17) into (3), we obtain the average channel capacity for SDM SU-MIMO without precoding as

$$
\begin{aligned}
C_{S U}^{O}= & \int_{0}^{\infty} d \gamma \log _{2}(1+\gamma) K[\underbrace{\int_{0}^{\gamma} \frac{2}{\gamma_{0}} e^{-\frac{2 x}{\gamma_{0}}}\left(1-e^{-\frac{2(\gamma-x)}{\gamma_{0}(1+x)}}\right) d x}_{\Theta}]^{K-1} \\
& \cdot \underbrace{\int_{0}^{\infty}\left[\frac{4}{\gamma_{0}^{2}(1+x)} \exp \left(-\frac{2\left(\gamma+x^{2}\right)}{\gamma_{0}(1+x)}\right)\right] d x}_{\Upsilon}
\end{aligned}
$$

where

$$
\boldsymbol{\Theta}=\frac{2}{\gamma_{0}} \underbrace{\int_{0}^{\gamma} e^{-\frac{2 x}{\gamma_{0}}} d x}_{\alpha}-\frac{2}{\gamma_{0}} \underbrace{\int_{0}^{\gamma} e^{-\frac{2 x^{2}+2 \gamma^{2}}{\gamma_{0}(1+x)}} d x}_{\beta}
$$

Therefore, $\boldsymbol{\Theta}=\frac{2}{\gamma_{0}} \alpha-\frac{2}{\gamma_{0}} \beta$, and $\alpha$ can be computed as

$\alpha=-\frac{\gamma_{0}}{2} \int_{0}^{\gamma} e^{-\frac{2 x}{\gamma_{0}}} d\left(-\frac{2 x}{\gamma_{0}}\right)=-\left.\frac{\gamma_{0}}{2} e^{-\frac{2 x}{\gamma_{0}}}\right|_{0} ^{\gamma}=\frac{\gamma_{0}}{2}\left(1-e^{-\frac{2 \gamma}{\gamma_{0}}}\right)$.

We derive $\beta$ by $u$-Substitution. Let $u=\gamma_{0}(1+x)$, we have $x=u / \gamma_{0}-1, d x=d u / \gamma_{0}$ and $x^{2}+\gamma^{2}=\left(\frac{u}{\gamma_{0}}-1\right)^{2}+\gamma^{2}=$ $\frac{u^{2}}{\gamma_{0}^{2}}-\frac{2 u}{\gamma_{0}}+1+\gamma^{2}$. Therefore,

$$
\begin{aligned}
\beta & =\frac{1}{\gamma_{0}} \int_{\gamma_{0}}^{\gamma_{0}(1+\gamma)} e^{-2 u^{-1}\left(\frac{u^{2}}{\gamma_{0}^{2}}-\frac{2 u}{\gamma_{0}}+1+\gamma^{2}\right)} d u \\
& =\frac{e^{4 / \gamma_{0}}}{\gamma_{0}} \int_{\gamma_{0}}^{\gamma_{0}(1+\gamma)} e^{b u+a u^{-1}} d u,
\end{aligned}
$$


where $b=-2 / \gamma_{0}^{2}$ and $a=-2(1+\gamma)$. Since $u=\gamma_{0}(1+x)$, we have $x=u / \gamma_{0}-1, d x=d u / \gamma_{0}$ and $x^{2}+\gamma=\left(\frac{u}{\gamma_{0}}-1\right)^{2}+\gamma=$ $\frac{u^{2}}{\gamma_{0}^{2}}-\frac{2 u}{\gamma_{0}}+1+\gamma, \Upsilon$ in (18) can be represented as

$$
\begin{aligned}
\Upsilon & =\int_{0}^{\infty} \frac{4}{\gamma_{0} u} \exp \left(-\frac{2}{u}\left[\frac{u^{2}}{\gamma_{0}^{2}}-\frac{2 u}{\gamma_{0}}+1+\gamma\right]\right) d x \\
& =\frac{4 e^{4 / \gamma_{0}}}{\gamma_{0}^{2}} \int_{0}^{\infty} u^{-1} \exp \left(-\frac{2 u}{\gamma_{0}^{2}}-\frac{2(1+\gamma)}{u}\right) d u
\end{aligned}
$$

According to [15, p. 144]

$$
\begin{aligned}
& \int_{0}^{\infty} e^{-(p x+q / x)} x^{-(a+1 / 2)} d x \\
& =\left(\frac{p}{q}\right)^{\frac{1}{2} a} \exp (-2 \sqrt{p q}) \sqrt{\frac{\pi}{p}} \sum_{n=0}^{\infty} \frac{(a-n)^{2 n}}{2^{n / 2}(2 \sqrt{p q})^{n}} .
\end{aligned}
$$

Let $a=1 / 2$, Eq. (21) becomes

$$
\begin{aligned}
& \int_{0}^{\infty} e^{-(p x+q / x)} x^{-1} d x \\
& \quad=\left(\frac{p}{q}\right)^{\frac{1}{4}} \exp (-2 \sqrt{p q}) \sqrt{\frac{\pi}{p}} \sum_{n=0}^{\infty} \frac{(1 / 2-n)^{2 n}}{2^{n / 2}(2 \sqrt{p q})^{n}} .
\end{aligned}
$$

Assigning $p=2 / \gamma_{0}^{2}, q=2(1+\gamma)$ in the above equation, $\boldsymbol{\Upsilon}$ in (20) can be derived as

$$
\begin{aligned}
\Upsilon= & \frac{4 e^{4 / \gamma_{0}}}{\gamma_{0}^{2}}\left[\gamma_{0}^{2}(1+\gamma)\right]^{-\frac{1}{4}} \exp \left(-\frac{4}{\gamma_{0}} \sqrt{1+\gamma}\right) \sqrt{\frac{\pi}{2}} \gamma_{0} \\
& \cdot \sum_{n=0}^{\infty} \frac{(1 / 2-n)^{2 n}}{2^{n / 2}\left(\frac{4}{\gamma_{0}} \sqrt{1+\gamma}\right)^{n}} .
\end{aligned}
$$

B. Derivation of (7) - the average channel capacity for $M U$ MIMO without precoding

For the SDM MU-MIMO without precoding, the average channel capacity has the form

$$
\begin{aligned}
C_{M U}^{O} & =\sum_{i} \int_{0}^{\infty} \log _{2}(1+x) \frac{n_{t}}{\gamma_{0}} e^{-\frac{n_{t} x}{\gamma_{0}}} K\left(1-e^{-\frac{n_{t} x}{\gamma_{0}}}\right)^{K-1} d x \\
& =\frac{n_{t} K}{\gamma_{0}} \sum_{i} \int_{0}^{\infty} \log _{2}(1+x) e^{-\frac{n_{t} x}{\gamma_{0}}}\left(1-e^{-\frac{n_{t} x}{\gamma_{0}}}\right)^{K-1} d x
\end{aligned}
$$

According to the binomial theorem [16, p. 25]

$$
(1-z)^{n}=\sum_{j=0}^{n}(-1)^{j} \frac{n !}{(n-j) ! j !} z^{j}
$$

we can derive

$$
e^{-\frac{n_{t} x}{\gamma_{0}}}\left(1-e^{-\frac{n_{t} x}{\gamma_{0}}}\right)^{K-1}=\sum_{j=0}^{K-1}(-1)^{j}\left(\begin{array}{c}
K-1 \\
j
\end{array}\right) e^{-\frac{(j+1) n_{t} x}{\gamma_{0}}}
$$

where the binomial coefficient is given by

$$
\left(\begin{array}{c}
K-1 \\
j
\end{array}\right)=\frac{(K-1) !}{(K-j-1) ! j !} .
$$

To solve the integral in (24), let us first consider

$$
\mathcal{A}=\int_{0}^{\infty} \log _{2}(1+x) e^{a_{j} x} d x
$$

where $a_{j}=-\frac{(j+1) n_{t}}{\gamma_{0}}$. The closed form expression of (27) can be derived as

$$
\begin{aligned}
\mathcal{A} & =\frac{1}{a_{j} \ln 2} \int_{0}^{\infty} \ln (1+x) d\left(e^{a_{j} x}\right) \\
& =\left.\frac{1}{a_{j} \ln 2} \ln (1+x) e^{a_{j} x}\right|_{0} ^{\infty}-\frac{1}{a_{j} \ln 2} \int_{0}^{\infty} e^{a_{j} x} d[\ln (1+x)] \\
& =-\frac{1}{a_{j} \ln 2} \int_{0}^{\infty} \frac{e^{a_{j} x}}{1+x} d x
\end{aligned}
$$

Equ. (28) is derived by following the fact that $\lim _{y \rightarrow \infty} \frac{\ln y}{e^{-c y}}=$ $0(c<0)$, and by assigning $u=\ln (1+x), v \stackrel{y \rightarrow \infty}{=} e^{a_{j} x}$, then performing integral by parts. According to [16, p. 337],

$$
\int_{0}^{\infty} \frac{e^{-\mu x}}{x+\beta} d x=-e^{\beta \mu} E_{i}(-\mu \beta), \quad \mu>0
$$

Assigning $\beta=1, \mu=-a_{j}$ in (29), the closed form of (28) can be obtained as

$$
\int_{0}^{\infty} \log _{2}(1+x) e^{a_{j} x} d x=\frac{e^{-a_{j}} E_{i}\left(a_{j}\right)}{a_{j} \ln 2},
$$

where the exponential integral function $E_{i}(x)$ is defined in (8).

Substituting (26) and (30) into (24), we can derive the average channel capacity for SDM MU-MIMO without precoding

$$
\begin{aligned}
C_{M U}^{O} & =\frac{n_{t} K}{\gamma_{0}} \sum_{i} \int_{0}^{\infty} \log _{2}(1+x) \sum_{j=0}^{K-1}(-1)^{j}\left(\begin{array}{c}
K-1 \\
j
\end{array}\right) e^{-\frac{(j+1) n_{t} x}{\gamma_{0}}} d x \\
& =\frac{n_{t} K}{\gamma_{0}} \sum_{i} \sum_{j=0}^{K-1}(-1)^{j}\left(\begin{array}{c}
K-1 \\
j
\end{array}\right) \int_{0}^{\infty} \log _{2}(1+x) e^{-\frac{(j+1) n_{t} x}{\gamma_{0}}} d x \\
& =\frac{n_{t} K}{\gamma_{0} \ln 2} \sum_{i} \sum_{j=0}^{K-1}(-1)^{j}\left(\begin{array}{c}
K-1 \\
j
\end{array}\right) \frac{e^{-a_{j}} E_{i}\left(a_{j}\right)}{a_{j}}
\end{aligned}
$$

where $a_{j}=-\frac{(j+1) n_{t}}{\gamma_{0}}$.

C. Derivation of (16) - channel capacity for systems with large number of antennas

For the systems where $n_{r}$ and/or $n_{t}$ is large, $\beta(i)$ is sufficiently large. Under such circumstances, we can utilize the series representation of the exponential function

$$
e^{x}=1+x+\frac{x^{2}}{2 !}+\frac{x^{3}}{3 !}+\ldots=\sum_{j=0}^{\infty} \frac{x^{j}}{j !} \approx \sum_{j=0}^{\beta(i)-1} \frac{x^{j}}{j !} .
$$

For a linearly precoded SDM MU-MIMO, the average channel capacity can be expressed as

$$
\begin{aligned}
& C_{M U}^{C} \simeq \frac{K}{\rho_{i} \tilde{\lambda}_{i}} \boldsymbol{\Phi}+\sum_{i=1}^{\eta} \frac{K}{\left(\rho_{i} \tilde{\lambda}_{i}\right)^{\beta(i)}(\beta(i)-1) !} \\
& \underbrace{\int_{0}^{\infty} \log _{2}(1+\gamma) \underbrace{\gamma^{\beta(i)-1} e^{-\frac{\gamma}{\rho_{1} \tilde{\lambda}_{i}}}\left[1-\sum_{j=0}^{\beta(i)-1} \frac{\gamma^{j}}{j !\left(\rho_{i} \tilde{\lambda}_{i}\right)^{j}} e^{-\frac{\gamma}{\rho_{i} \tilde{\lambda}_{i}}}\right]^{K-1}}_{\boldsymbol{\Psi}(\gamma)} d \gamma}_{\boldsymbol{\Omega}},
\end{aligned}
$$


where $\eta=n_{t}=n_{r}$ and the ordered eigenvalues of the complex central Wishart matrix $\mathbf{H} \mathbf{H}^{H}$ is $\lambda_{1} \geq \lambda_{2} \geq \cdots \geq \lambda_{\eta}$.

When $i=\eta, \beta(\eta)=1$, following the same procedure as shown in Section III-A for SDM MU-MIMO without precoding, we have

$$
\frac{K}{\rho_{i} \tilde{\lambda}_{i}} \boldsymbol{\Phi}=\frac{K}{\rho_{\eta} \tilde{\lambda}_{\eta}} \frac{1}{\ln 2} \sum_{j=0}^{K-1}(-1)^{j}\left(\begin{array}{c}
K-1 \\
j
\end{array}\right) \frac{e^{-d_{j}} E_{i}\left(d_{j}\right)}{d_{j}},
$$

where $d_{j}=-\frac{(j+1)}{\rho_{\eta} \lambda_{\eta}}$. With binomial expansion, we have

$$
\begin{aligned}
& \boldsymbol{\Psi}(\gamma)=\gamma^{\beta(i)-1} e^{-\frac{\gamma}{\rho_{i} \tilde{\lambda}_{i}}}\left[1-\sum_{j=0}^{\beta(i)-1} \frac{\gamma^{j}}{j !\left(\rho_{i} \tilde{\lambda}_{i}\right)^{j}} e^{-\frac{\gamma}{\rho_{i} \tilde{\lambda}_{i}}}\right]^{K-1} \\
& =\gamma^{\beta(i)-1} \sum_{n=0}^{K-1}(-1)^{n} \frac{(K-1) !}{(K-1-n) ! n !} \\
& \quad\left(\sum_{j=0}^{\beta(i)-1} \frac{\gamma^{j}}{j !\left(\rho_{i} \tilde{\lambda}_{i}\right)^{j}}\right)^{n} e^{-\frac{(n+1) \gamma}{\rho_{i} \tilde{\lambda}_{i}}} \\
& =\gamma^{\beta(i)-1} \sum_{n=0}^{K-1} c_{n}\left(\sum_{j=0}^{\beta(i)-1} \frac{\gamma^{j}}{j !\left(\rho_{i} \tilde{\lambda}_{i}\right)^{j}}\right)^{n} e^{-\frac{(n+1) \gamma}{\rho_{i} \tilde{\lambda}_{i}}}
\end{aligned}
$$

where $c_{n}$ is given by (15).

When $\beta(i)$ is large, Equ. (33) can be approximated by

$$
\begin{aligned}
\boldsymbol{\Psi}(\gamma) & =\sum_{n=0}^{K-1} c_{n} \gamma^{\beta(i)-1}\left(\sum_{j=0}^{\beta(i)-1} \frac{\left[\gamma /\left(\rho_{i} \tilde{\lambda}_{i}\right)\right]^{j}}{j !}\right)^{n} e^{-\frac{(n+1) \gamma}{\rho_{i} \tilde{\lambda}_{i}}} \\
& \approx \sum_{n=0}^{K-1} c_{n} \gamma^{\beta(i)-1} e^{\frac{\gamma n}{\rho_{i} \tilde{\lambda}_{i}}} e^{-\frac{(n+1) \gamma}{\rho_{i} \tilde{\lambda}_{i}}}=\sum_{n=0}^{K-1} c_{n} \gamma^{\beta(i)-1} e^{-\frac{\gamma}{\rho_{i} \tilde{\lambda}_{i}}}
\end{aligned}
$$

Therefore,

$$
\begin{aligned}
\boldsymbol{\Omega} & =\int_{0}^{\infty} \log _{2}(1+\gamma) \boldsymbol{\Psi}(\gamma) d \gamma \\
& =\int_{0}^{\infty} \log _{2}(1+\gamma) \sum_{n=0}^{K-1} c_{n} \gamma^{\beta(i)-1} e^{-\frac{\gamma}{\rho_{i} \tilde{\lambda}_{i}}} d \gamma \\
& =\frac{1}{\ln 2} \sum_{n=0}^{K-1} c_{n} \mathcal{I}_{\beta(i)}\left(\frac{1}{\rho_{i} \tilde{\lambda}_{i}}\right) \\
& =\frac{1}{\ln 2} \sum_{n=0}^{K-1}(-1)^{n} \frac{(K-1) !}{(K-1-n) ! n !} \mathcal{I}_{\beta(i)}\left(\frac{1}{\rho_{i} \tilde{\lambda}_{i}}\right)
\end{aligned}
$$

According to $(31), C_{M U}^{C} \approx \sum_{i=1}^{\eta-1} \frac{K}{\left(\rho_{i} \tilde{\lambda}_{i}\right)^{\beta(i)}(\beta(i)-1) !} \boldsymbol{\Omega}+$ $\frac{K}{\rho_{\eta} \tilde{\lambda}_{\eta}} \Phi$.

\section{REFERENCES}

[1] 3GPP TR 25.814 V7.0.0, "Physical Layer Aspects for Evolved UTRA," Tech. Rep., June 2006.

[2] N. Wei, A. Pokhariyal, T. B. Sørensen, T. E. Kolding, and P. E. Mogensen, "Performance of MIMO with Frequency Domain Packet Scheduling in UTRAN LTE downlink," in IEEE VTC, Apr. 2007, pp. 1177-1181.

[3] J. Zhang, Y. Wu, M. Xu, and J. Wang, "Linear transmitter precoding design for downlink of multiuser MIMO system," Electronics Letters, vol. 41, no. 14, July 2005.

[4] Q. H. Spencer, A. L. Swindlehurst, and M. Haardt, "Zero-forcing Methods for Downlink Spatial Multiplexing in Multiuser MIMO Channels," Transactions on Singal Processing, vol. 52, no. 2, pp. 461-471, Feb. 2004.
[5] A. G. Kogiantis, N. Joshi, and O. Sunay, "On Transmit Diversity and Scheduling in Wireless Packet Data," in IEEE ICC, 2001, pp. 2433-2437.

[6] L. T. Berger, T. E. Kolding, J. R. Moreno, P. Ameigeiras, L. Schumacher, and P. E. Mgensen, "Interaction of transmit diversity and proportional fair scheduling," in IEEE VTC, spring 2003, pp. 2423-2427.

[7] R. Gozali, R. M. Buehrer, and B. D. Woerner, "The impact of multiuer diversity on space time block coding," IEEE Commun. Letters, vol. 7, no. 5, pp. 213-215, May 2003.

[8] T. Park, O.-S. Shin, and K. B. Lee, "Proportional Fair Scheduling for Wireless Communication with Multiple Transmit and Receive Antennas," in IEEE VTC, Florida, USA, October 2003, vol. 3, pp. 1573 - 1577.

[9] V. K. N. Lau, "Proportional fair space-time scheduling for wireless communications," IEEE Trans. Commun., vol. 53, no. 8, pp. 1353-1360, Aug. 2005.

[10] D. P. Palomar, J. M. Cioffi, and M. A. Lagunas, "Joint Tx-Rx Beamforming Design for Multicarrier MIMO Channels: A Unified Framework for Convex Optimization," IEEE Transaction on Signal Processing, vol. 51, no. 9, pp. 2381-2401, Sept. 2003.

[11] W. C. Y. Lee, "Estimate of channel capacity in Rayleigh fading enviroment," IEEE Trans. on Veh. Technol., vol. 39, pp. 187-190, Aug. 1990

[12] A. Goldsmith, Wireless Communications, Cambridge University Press, 2005.

[13] Z. Lin, P. Xiao, B. Vucetic. "SINR Distribution for LTE Downlink Multiuser MIMO Systems," Proc. IEEE ICASSP'09, pp. 2833-2836, April 2009.

[14] M. Alouini and A. Goldsmith, "Capacity of Rayleigh fading channels under different adaptive transmission and diversity-combining techniques," IEEE Trans. Veh. Tech.,, vol. 48, no. 4, pp. 1165-1181, July 1999.

[15] D. Dierens de Haan, Nouvelles Tables, d'integrales definies, Hafner Publishing Company, 1867.

[16] I. Gradshteyn and I. Ryzhik, Tables of Integrals, Serials, and Products, Academic Press, 6th edition, 2000. 